\title{
PLASTICITY MODELLING IN PM STEELS
}

\section{Andersson and V. Angelopoulos}

\begin{abstract}
Simulations are continuously becoming more and more important to predict the behaviour of materials, components and structures. Porous materials, such as PM, put special demands on the material models used. This paper investigates the application of the Gurson material model to PM steels. It is shown how the model can be calibrated to material data. The results are also applied to an indentation test, where it's demonstrated that experimental results can be reproduced with some accuracy. Limitations of the model, and the potential to use more advanced material models are also discussed.
\end{abstract}

Kewords: PM steels, plasticity, material modelling, Gurson model

\section{INTRODUCTION}

Simulations are continuously becoming more and more important to predict the behaviour of materials, components and structures. By applying advanced material modelling as a supplement to experiments, it is often possible to shorten lead times during development considerably. One example is surface densification of sintered gears through gear rolling, see for instance [1-3]. By using finite element simulations it is possible to simulate the process and optimize for instance the tool geometry or the material stock on the work pieces.

However, the accuracy of the simulations will depend on how well the material model predicts the material behaviour. Classic metal plasticity models typically rely on von Mises plasticity, and results in zero volumetric strain. This is clearly not correct for densification of PM steels, and special plasticity models for porous materials should be used instead.

One model that is often used is the Gurson plasticity model [4], that is also found in some commercial finite element software such as Abaqus [5]. The Gurson yield criterion combines the von Mises stress with the hydrostatic stress, while accounting for different porosity levels, into a model suitable for porous materials. Fig. 1 illustrates the yield surfaces for different porosity levels, note that for zero porosity $(\mathrm{f}=0)$ the model is identical to classic von Mises plasticity.

The purpose of this paper is first to investigate how the necessary input to the Gurson model can be calibrated from tensile tests on PM materials. Next, to simulate surface densification, indentation tests are made where a steel ball is pressed into the surface of a PM material. The experimental indentation tests are then compared to calculated ones, testing the application of the Gurson model. 


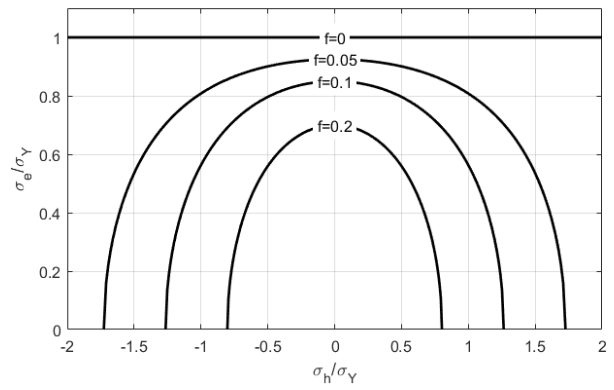

Fig.1. Gurson yield surfaces for different porosity levels, von Mises stress versus hydrostatic

pressure.

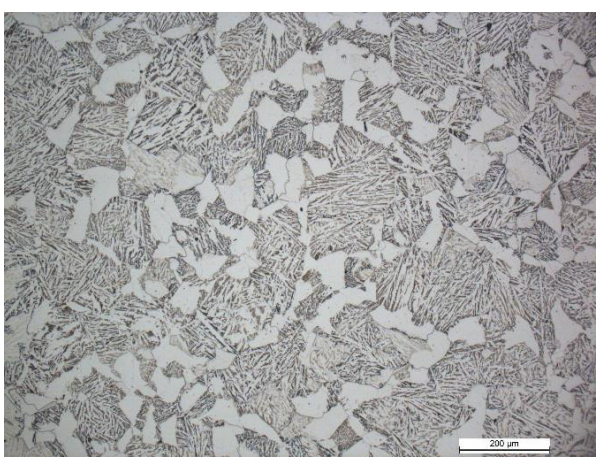

Fig.2. Example of micro structure, HIP:ed material

\section{MATERIAL}

The material used for this investigation is Astaloy ${ }^{\mathrm{TM}} 85 \mathrm{Mo}+0.3 \% \mathrm{C}$ UF4+0.6\%Lube E from Höganäs AB. Test bars were compacted to different densities, see further below, and sintered at $1120^{\circ} \mathrm{C}$ for $45 \mathrm{~min}$ in $90 / 10 \mathrm{~N}_{2} / \mathrm{H}_{2}$. Some test bars were also HIP:ed to produce a pore free structure, these were double pressed and double sintered prior to HIP in order to remove all inter connected porosity. HIP:ing was done at $1150^{\circ} \mathrm{C}$ and 1000 bar for 180 min. In all cases the resulting micro structure was a mix of bainite and ferrite, typical for this material. An example of the HIP:ed micro structure is shown in Fig.2.

\section{CALIBRATING THE GURSON MODEL}

To use the Gurson model for simulations it's necessary to provide the hardening of the matrix material to the model. In many cases it's easier to do the test on the porous material. Therefore it's also necessary to be able to calibrate the matrix, given the hardening curve from a standard PM tensile test. In the following the equations for the Gurson model are derived for the uniaxial case, and an integration scheme presented to be used for the calibration of the model.

The yield surface for the Gurson model [4] is given by:

$\Phi=\left(\frac{q}{\sigma_{y}}\right)^{2}+2 q_{1} f \cosh \left(\frac{-3 q_{2} p}{2 \sigma_{y}}\right)-\left(1+q_{3} f^{2}\right)$

Here $f$ is the volume fraction of pores, $q$ denotes the von Mises stress and $p$ the hydrostatic pressure according to:

$q=\sigma_{v M}=\sqrt{\frac{3}{2} s_{i j} s_{i j}}$

$s_{i j}=\sigma_{i j}+p \delta_{i j}$

$p=-\frac{1}{3} \sigma_{k k}$

Where standard index notation is applied. It should also be noted that the yield surface was modified with the constants $q_{i}$ according to Tvergaard [5]. In addition to the yield surface 
evolution laws, see for instance [5] or [6], are given for the porosity and the matrix material according to:

$$
\begin{aligned}
& d f=(1-f) d \varepsilon_{k k}^{p} \\
& \sigma_{i j} d \varepsilon_{i j}^{p}=(1-f) \sigma_{y} d \varepsilon_{m}^{p}
\end{aligned}
$$

where $\varepsilon_{i j}^{p}$ is the plastic strain tensor and $\sigma_{y}$ and $\varepsilon_{m}{ }^{p}$ yield stress and plastic strain of the matrix material, with $\sigma_{y}=f\left(\varepsilon_{m}^{p}\right)$. Isotropic hardening is assumed.

Assuming the associated flow rule, the plastic strain increment is given as:

$$
d \varepsilon_{i j}^{p}=d \lambda \frac{\partial \Phi}{\partial \sigma_{i j}}=d \lambda \cdot\left(\frac{-1}{3} \frac{\partial \Phi}{\partial p} \delta_{i j}+\frac{3}{2 q} \frac{\partial \Phi}{\partial q} s_{i j}\right)
$$

with

$$
\begin{aligned}
& \frac{\partial \Phi}{\partial p}=\frac{-3 q_{1} q_{2} f}{\sigma_{y}} \sinh \frac{-3 q_{2} p}{2 \sigma_{y}} \\
& \frac{\partial \Phi}{\partial q}=\frac{2 q}{\sigma_{y}^{2}}
\end{aligned}
$$

From which it is also clear that the model includes plastic compressibility.

Furthermore, for the pure uniaxial case the following equations are obtained:

$$
\begin{aligned}
& \Phi=\left(\frac{\sigma}{\sigma_{y}}\right)^{2}+2 q_{1} f \cosh \left(\frac{q_{2} \sigma}{2 \sigma_{y}}\right)-\left(1+q_{3} f^{2}\right)=0 \\
& \sigma \cdot d \varepsilon_{p}=\hat{\rho} \sigma_{y} d \varepsilon_{m}^{p}
\end{aligned}
$$

with $\sigma$ denoting the uniaxial stress, $\varepsilon_{p}$ the uniaxial plastic strain, and combining the equations above the following equation is obtained:

$$
d \hat{\rho}=\frac{-3 q_{1} q_{2} \hat{\rho}(1-\hat{\rho}) \sigma_{y} \sinh \frac{q_{2} \sigma}{2 \sigma_{y}}}{2 \sigma+q_{1} q_{2}(1-\hat{\rho}) \sigma_{y} \sinh \frac{q_{2} \sigma}{2 \sigma_{y}}} \cdot d \varepsilon_{p}
$$

Here the volume fraction of pores, $f$, was replaces with the relative density according to:

$\hat{\rho}=\frac{\rho}{\rho_{0}}=1-f$

The procedure used to integrate the equations is to use a simple Euler forward scheme with $d \varepsilon_{p} \rightarrow \Delta \varepsilon_{p}$. The procedure then becomes:

$$
\begin{aligned}
& \text { select } \Delta \varepsilon_{\mathrm{p}, \mathrm{i}} \\
& \text { calculate } \varepsilon_{\mathrm{p}, \mathrm{i}+1}=\varepsilon_{\mathrm{p}, \mathrm{i}}+\Delta \varepsilon_{\mathrm{p}, \mathrm{i}} \\
& \text { calculate } \varepsilon_{\mathrm{m}, \mathrm{i}+1}^{\mathrm{p}} \text { using equation (11) } \\
& \text { calculate } \rho_{\mathrm{i}+1} \text { using equation (12) } \\
& \text { solve } \Phi=0
\end{aligned}
$$

with index $i$ denoting the current step. Note that in step 5 either $\sigma$ can be solved given the matrix behavior $\sigma_{y}$ or the opposite if the matrix is the unknown. This way either the hardening of the porous material can be calculated, or the hardening curve of the matrix material. 
In the original formulation of the Gurson yield surface the parameters $q_{i}=1$, whereas Tvergaard [6] suggested that they should be taken as $q_{1}=1.5, q_{2}=1$ and $q_{3}=2.25$, which he showed improved the predictions from the model. Other values for $q_{i}$ are also possible, but these two variants are used here.

From the measured tensile curves, the matrix material behavior was calculated using the above procedure. The results are given in Fig. 3. As expected the model predicts a matrix material that has a higher yield strength than the PM material itself. It can be observed from Fig. 3a that the Tvergaard choice for $\mathrm{q}_{\mathrm{i}}$ yield a harder material compared to $\mathrm{q}_{\mathrm{i}}=1$. Note that the figure only shows the plastic part of the tensile curve.
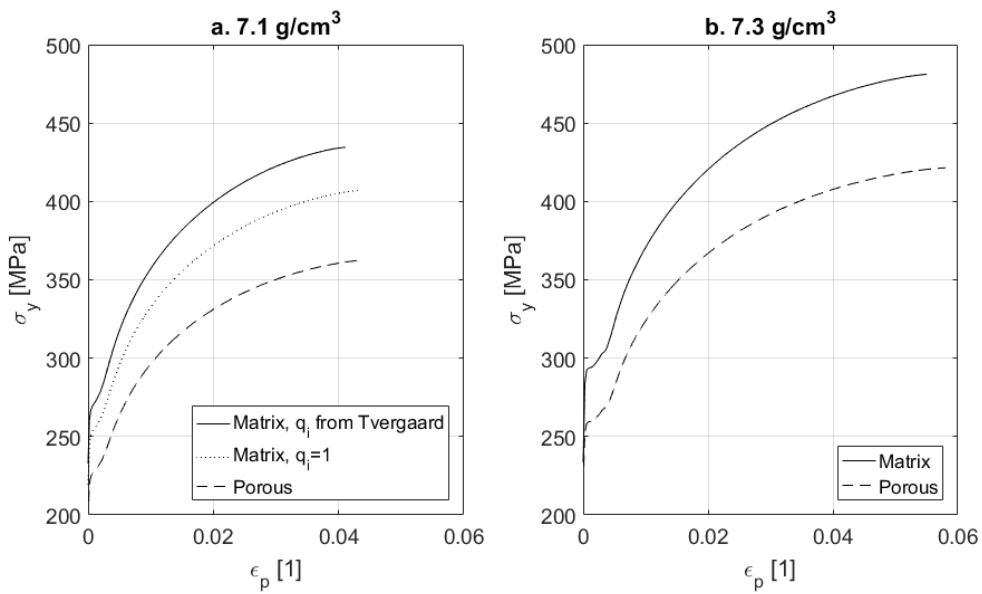

Fig.3. Tensile curves, with calibrated matrix materials for density levels of a. $7.1 \mathrm{~g} / \mathrm{cm}^{3}$ and b. $7.3 \mathrm{~g} / \mathrm{cm}^{3}$

Fig. 4 summarizes the two calibrated curves and the tensile curve obtained from HIP:ed test bars. As can be seen the two different densities yield similar, but not identical matrix materials. Also, the HIP:ed material shows a significantly harder material compared to the calibrated curves. For the simulations the hardening behavior for $\rho=7.1 \mathrm{~g} / \mathrm{cm}^{3}$ will be used, as well as the HIP:ed curve.

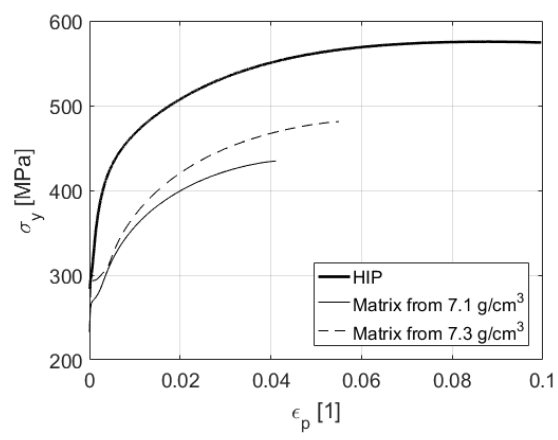

Fig.4. Calibrated matrix materials versus HIP test bars.

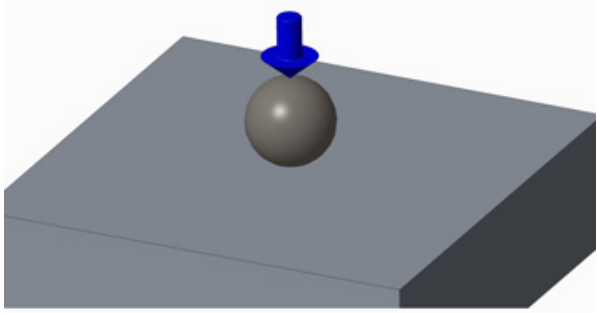

Fig.5. Test setup for indentation tests 


\section{INDENTATION TESTS}

As mentioned above, one of the target applications where the plasticity models play an important role is surface densification of PM gears through rolling. However, since the gear rolling process is rather complex, it makes sence to also test the material models on simpler load cases. In this case it was decided to do an indentation test with a steel bearing ball onto the surface of a PM material, as illustrated in Fig. 5. This test produces a local indentation on the surface, that can later be measured and compared to simulations.

For the results presented here, a stell ball with a diamter of $15 \mathrm{~mm}$ was pressed with $5 \mathrm{kN}$ onto the surface of a bar with dimensions $120 \times 30 \times 30 \mathrm{~mm}^{3}$ made of PM steels with densities of 7.1 and $7.2 \mathrm{~g} / \mathrm{cm}^{3}$. After the tests the indentation mark was measured using a profilometer, giving the depth of the indent as a function of the distance from the centre.

A finite element model was also developed of the geometry. An overview of the model is shown in Fig. 6 . The ball was modelled as an elastic half sphere with $\mathrm{E}=206 \mathrm{GPa}$ and $v=0.3$, and the PM plate modelled using the Gurson model and the hardening curves from above. Axisymmetric elements were used due to the symmetry of the setup. The load was applied to the sphere as a uniform pressure corresponding to $5 \mathrm{kN}$. Contact conditions were applied between the bodies.

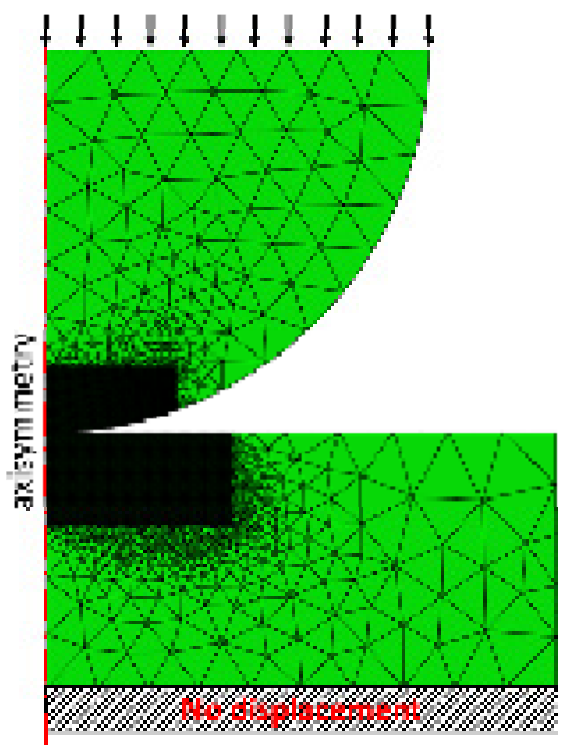

Fig.6. Overview of finite element model.
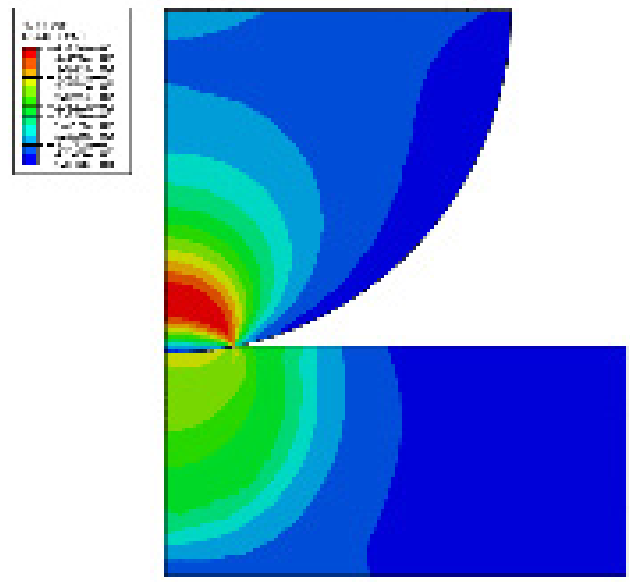

Fig.7. von Mises stress at max load, case I.

To investigate the influence of different material models four simulations were done, according to Tab. 1. For the simulations different base tensile tests were used to derive the matrix material, different parameters were applied to the Gurson model and two different start densities were used for the base plae. From the simulations the indentation profile, and density profile after unloading were determined, and the former compared to the experiments. Fig. 7 also shows the von Mises stresses at max indentation force. 
Tab.1. Overview of simulations.

\begin{tabular}{|c|c|c|c|}
\hline Case & Tensile test bar & Density plate & Gurson parameters \\
\hline I & $7.1 \mathrm{~g} / \mathrm{cm}^{3}$ & $7.1 \mathrm{~g} / \mathrm{cm}^{3}$ & $\{1.5,1,2.25\}$ \\
\hline II & HIP:ed & $7.1 \mathrm{~g} / \mathrm{cm}^{3}$ & $\{1.5,1,2.25\}$ \\
\hline III & $7.1 \mathrm{~g} / \mathrm{cm}^{3}$ & $7.1 \mathrm{~g} / \mathrm{cm}^{3}$ & $\{1,1,1\}$ \\
\hline IV & $7.1 \mathrm{~g} / \mathrm{cm}^{3}$ & $7.2 \mathrm{~g} / \mathrm{cm}^{3}$ & $\{1.5,1,2.25\}$ \\
\hline
\end{tabular}

Fig. 8 shows the densified layer after the indentation and Fig. 9 the density profile at the centre axis, both for case I. It can be observed that the simulations predict a max relative densification of around $97 \%$ at the surface. The maximum is found slightly off centre. At half a mm below the surface the relative density is at $94 \%$ compared to the initial relative density of $90.5 \%$.

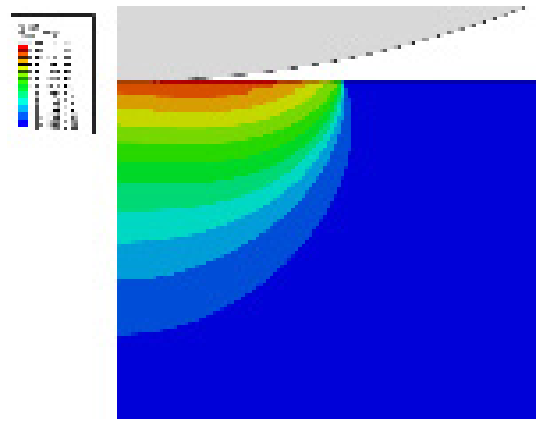

Fig.8. Relative density, case I.

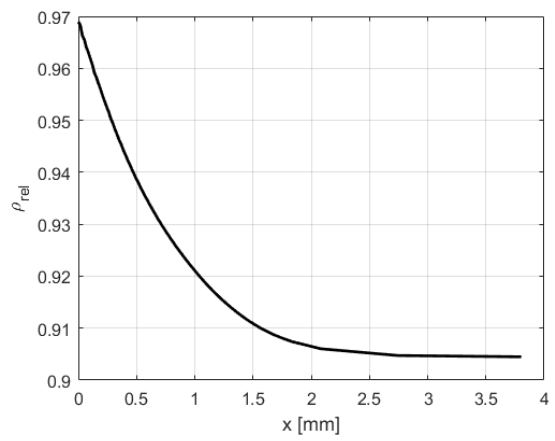

Fig.9. Density profile along centre axis, case I.

Unfortunately the experimental densification depth has not been measured, but to test the simulations against the experiments the indentation profiles can be compared. Fig. 10 shows the experimental profiles compared to the simulated for the different cases. It can be observed that the simulations all predict a more shallow denification depth compared to the experiments. Best results were obtained when the matrix material was based on the tensile test bars with $\rho=7.1 \mathrm{~g} / \mathrm{cm}^{3}$ PM material rather than the HIP:ed material. Case I and IV gave $11-13 \%$ less indentation depth compared to the experiments, whereas case II were at $34 \%$ less depth. There is some influence of the choice of the $q_{i}$ parameters, where the Tvergaard choise gave slightly better results, but not a big difference.

The width of the indentation zone is rougly the same in both simulations and experiments, except for case II, which is based on a material with a higher yield strength (the HIP:ed case). It is also worth noticing that the simulated profiles show a material transport going up at the edge on the contact zone, forming a ridge, whereas the simulations rather go down in the same are. 

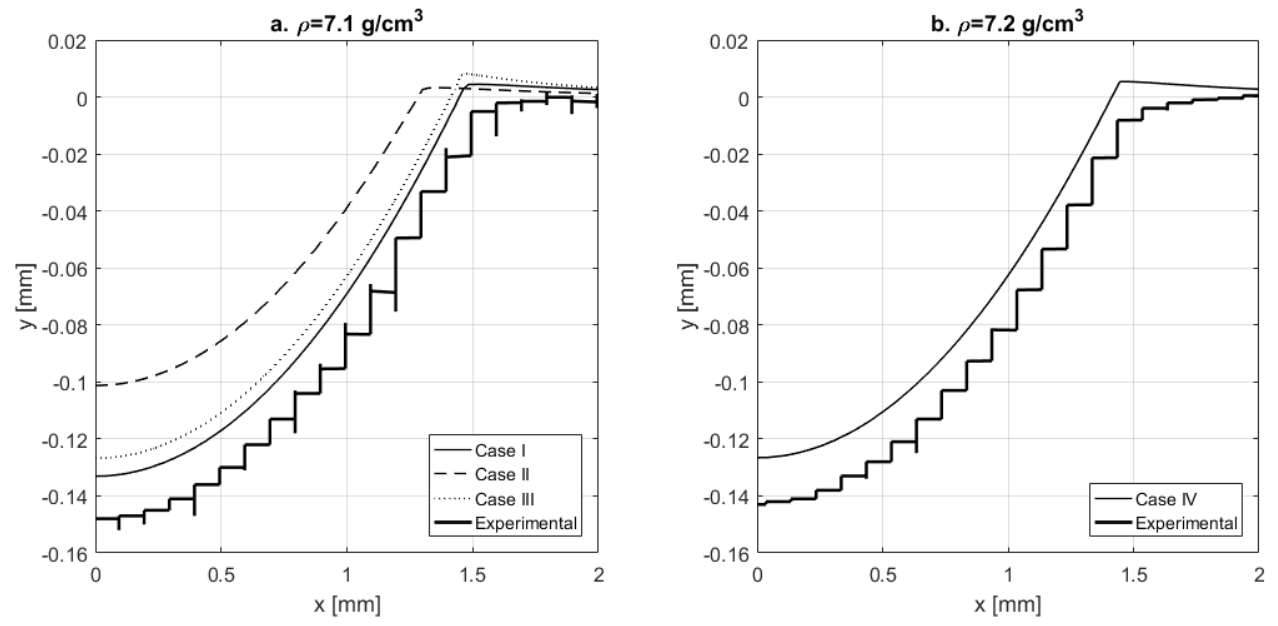

Fig.10. Densified profiles, a. $\rho_{\text {base }}=7.1 \mathrm{~g} / \mathrm{cm}^{3}$, b. $\rho_{\text {base }}=7.2 \mathrm{~g} / \mathrm{cm}^{3}$..

\section{DISCUSSION}

It is clear that different starting points for the tensile test yield different behaviour of the matrix material, see Fig. 4. Starting with tensile tests of PM steels at two different densities yield similar results, whereas the HIP:ed material displays a significantly higher yield strength for the matix. Ideally the Gurson model should give similar results for all cases, and the difference could be interpreted as the model is not able to cope with materials spanning over larger ranges of porosity.

It could be possible to improve the results somewhat by optimizing the $q_{i}$ parameters to modify the yield surface, and the influence from the pore fraction $f$. One drawback of this is that the otimization process would be more complicated, and that the end result need to be verified against more experimental data to make sure the result can be extrapolated. It should also be noted that the two different choices for $q_{i}$ tested here yielded very similar results on the indentation test.

Another drawback of the Gurson model as used in this study is that it does not take the change of elastic modulus with changing porosity into account. It is well known that the elastic modulus of a PM steel at $7.1 \mathrm{~g} / \mathrm{cm}^{3}$ is roughly $70 \%$ of a solid steel, and no easy method was found that allowed this effect to be included in the simulation. It will depend on the balance between elastsic and plastic strains if this effect will be important for the results and vary from case to case.

For the indentation simulations for instance, it was investigated what will happen if the elastic modulus is set to solid steel, compared to PM and the resulting difference in max indentation was found to be less than one percent.

Generally all simulations in this study showed less calculated indentation depth than the corresponding experiments. A difference in the material flow on the edge of the contact zone was alo found, where the simulations predicted a ridge that was not seen in the experimental results.

Using the tensile test results for PM materials with densities close to the material used for the indentation tests yielded the best results with a difference in max indentaion of $11-13 \%$. Since this result was obtained without further tweeking the parameters for the indentation tests it should be considered reasonably good. Small differences in for instance 
carbon content and cooling rate between tensile bars and indentation block may also explain part of the difference.

It would also be of interest to compare the densification profiles in the simulations with the experimental ones, unfortunately these experimental data are not available at this stage. One observation that can be made is that the max densification of the surface predicted by the simulations is around $97 \%$ relative density. This was surpricing since it is expected that the densification at the surface should be more or less $100 \%$.

As mentioned above, one big advantage with the Gurson model is that it is readily available in some commercial software, which makes it more accessible compared to alternative models. However, there are more advanced material models for porous metal plasticity such as the one suggested by Ponte Castañeda and Zaidman, [7]. This model contains a more accurate description of for instance the evaluation of the porosity and has been demonstrated to yield better results for gear rolling simulations, [1].

\section{CONCLUSIONS}

A procedure to calibrate the matrix material for the Gurson model was developed and applied to tensile test data. It was found that PM tensile curves from materials with different densities yield similar, but not identical matrix materials. However, tensile data for HIP:ed, fully dense material shows a higher yield strength.

Furthermore it was found that simulation of an indentation test tended to under estimate the indentation depth compared to experimental results. Best results were obtained if the matrix material was calibrated from tensile curves on material similar in density to the ones used for the indentation tests. Further calibration of the material parameters can be used to improve the results. Selection of the $q_{i}$ factors in the Gurson model seems to play less role.

One of the advantages of the Gurson model is that it is readily available in commercial finite element codes, which makes it easy to use. However, since there are limitations in the model, more advanced models might be necessary to accurately perform for instance gear rolling simulations.

\section{REFERENCES}

[1] Angelopoulos, V., Andersson, M. In: Proceedings of the JSME International conference on motion and power transmission. Vol. 2, 2017, p. 383

[2] Klocke, F. et.al.: Production Engineering, vol. 1, 2007, no. 2, p. 113

[3] Yazici, BA. et.al.: Powder Metallurgy, vol. 51, 2008, no. 3, p. 211

[4] Gurson, AL.: Journal of Engineering Materials and Technology, vol. 99, 1977, p. 2

[5] Abaqus

[6] Tvergaard, V.: International Journal of Fracture, vol. 17, 1981, no. 4, p. 389

[7] Ponte Castañeda, P., Zaidman, M.: Journal of the Mechanics and Physics of Solids, vol. 42, 1994, no. 9, p. 1459 